\title{
Late Solitary Acrometastasis of the Hands as Unusual Metastatic Site of Renal Cell Carcinoma: A Case Report and Literature Review
}

Won Hoon Song ${ }^{1}$, Sung Han Kim ${ }^{2}$, Weon Seo Park ${ }^{3}$, Sun Ho Kim ${ }^{4}$, June Hyuk Kim ${ }^{5}$, Jae Young Joung ${ }^{2}$, Ho Kyung Seo ${ }^{2}$, Kang Hyun Lee ${ }^{2}$ and Jinsoo Chung ${ }^{\star}$ ${ }^{1}$ Department of Urology, Seoul National University Hospital, Seoul

${ }^{2}$ Department of Urology, Center for Prostate Cancer, National Cancer Center, Goyang, Korea

${ }^{3}$ Department of Pathology, Center for Prostate Cancer, National Cancer Center, Goyang, Korea

${ }^{4}$ Department of Radiology, Center for Prostate Cancer, National Cancer Center, Goyang, Korea

${ }^{5}$ Department of Orthopedics, Center for Prostate Cancer, National Cancer Center, Goyang, Korea

*Corresponding author: Jinsoo Chung, Department of Urology, Center for Prostate Cancer, National Cancer Center, Goyang, Korea, Tel: +82-31-920-1676; Fax: +82-31-920-1790; E-mail: cjs5225@ncc.re.kr

Received date: Oct 15, 2015; Accepted date: Dec 02, 2015; Published date: Dec 04, 2015

Copyright: (c) 2015 Song WH, et al. This is an open-access article distributed under the terms of the Creative Commons Attribution License, which permits unrestricted use, distribution, and reproduction in any medium, provided the original author and source are credited.

\begin{abstract}
Skeletal metastases from renal cell carcinoma (RCC) are common, but its metastasis to the digital extremities is rare. The lesion presents diagnostic and therapeutic challenges because of the mimicry of other benign skeletal diseases resulting in difficulties in prompt recognition of diagnosis. We here report a 62 year-old male patient with RCC metastatic to a metacarpal bone presenting with pain and swelling of left hand after 8 years' follow-up at stable disease without any recurrences. The pathological cytology from the swelling site revealed findings consistent with metastatic clear cell carcinoma.
\end{abstract}

Keywords: Acrometastasis; Metacarpal bone; Hand; Renal cell carcinoma; Metastasis

\section{Introduction}

Acrometastasis are metastases located distal to the elbow and knee with a rate of $0.1 \%$ of all metastatic bone lesions [1-3]. Hand metastases of renal cell carcinoma (RCC) are rare and represent an uncommon site of recurrence $[1,2]$. The lesion presents diagnostic and therapeutic challenges because of the mimicry of other benign skeletal diseases resulting in difficulties in prompt recognition of diagnosis [2]. Clinical awareness of unusual sites of metastatic dissemination is a must in the face of increasing cancer survivorship. Acrometastasis portends a poor prognosis with limited survival, and optimal integration of the best supportive care is mandatory. Here, we report the case of a patient with RCC metastasis to the left third metacarpal bone treated with surgical resection of the acrometastasis and followup with stable disease without any further therapy.

\section{Case Report}

We report a case of 62-year old Asian man with eight years' diseasefree state of clear cell RCC staged T1bN0M0 after radical nephrectomy, representing a recently developed painful left hand during a routine follow-up of RCC. In his first postoperative year's follow-up, multiple suspicious lesions on the right parietal bone, left periorbital bone and left proximal femur without any symptoms or signs on bone scan were found to perform a targeted bony biopsy of the zygoma and magnetic resonance imaging of the left proximal femur finally revealed to diagnose polyostotic fibrous dysplasia. A thorough inquiry into the patient's background revealed a family history of systemic Behçet disease, and a history of multiple traumas. The patient was followed-up regularly without further treatment.
When the patient presented with painful swelling in the left hand at the eight-year postoperative follow-up, an osteolytic, expansile bony lesion with osteosclerotic rim on the left third metacarpal head and right forearm on simple X-ray correlated with a high-uptake lesion that was evident on a bone scan, indicating metastasis from RCC (Figure 1). (We did not perform biopsy prior to surgery). Subsequently, orthopedic curettage surgery of the mass lesion was performed successfully to confirm bone metastasis from clear cell RCC tissue (Figure 2). The patient had full recovery without complications. Three months postoperatively, a PET-CT showed no further, newly suspicious metastatic lesions, and no changes to the multiple known bone lesions. The patient refused to undergo further additional systemic therapy with a stable disease state until postoperative six months.

\section{Discussion}

Renal cell carcinoma (RCC) is well known for its unpredictable presentation, mode of spread, and high propensity for early metastasis and the overall incidence of metastatic tumors in the bone has been estimated at $30 \%$ or greater [4]. Metastases to the bones of the hands and feet (acrometastasis) represent about $0.1 \%$ of all bone metastasis and only $10-18.4 \%$ of acrometastases originate from RCC [2,5]. RCC is the most comon primary in foot metastases (18.2\%), equal in incidence to secondary lesions from large bowel. Renal carcinomatous metastases are also the third most common cause of metastases to the hand, constituting $18.4 \%$ of the total [2]. As compared with women, men are reportedly twice as likely to be affected [6]. And the dominant hand was more often affected [2]. They postulated this was because the dominant hand receives a larger amount of blood supply, and is more likely to undergo injuries than the non-dominant one. The following common sites of involvement in the hands have been reported: distal phalanx (74 lesions), metacarpals (56 lesions), proximal phalanx (26 lesions), and middle phalanx (16 lesions) [6]. 
Citation: Song WH, Kim SH, Park WS, Kim SH, Kim JH, et al. (2015) Late Solitary Acrometastasis of the Hands as Unusual Metastatic Site of Renal Cell Carcinoma: A Case Report and Literature Review. J Cytol Histol S3: 027. doi:10.4172/2157-7099.S3-027
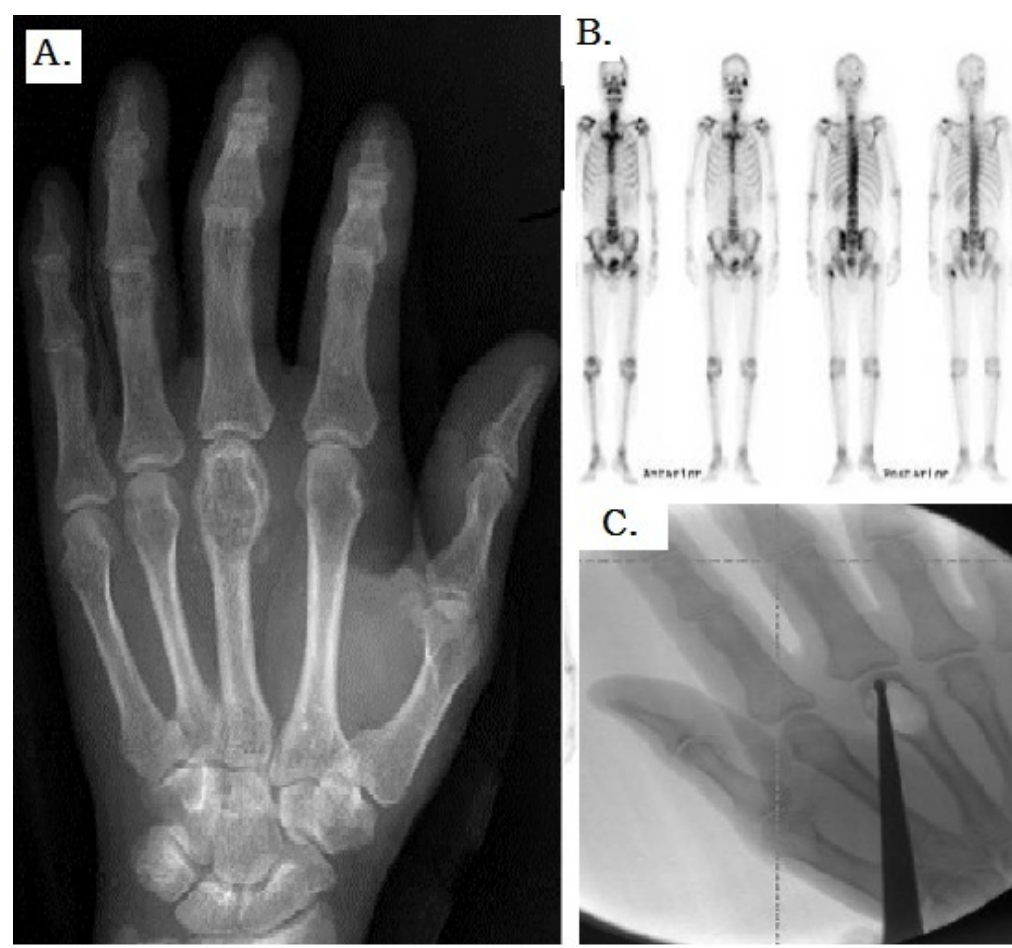

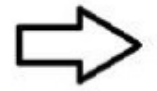

8 years after

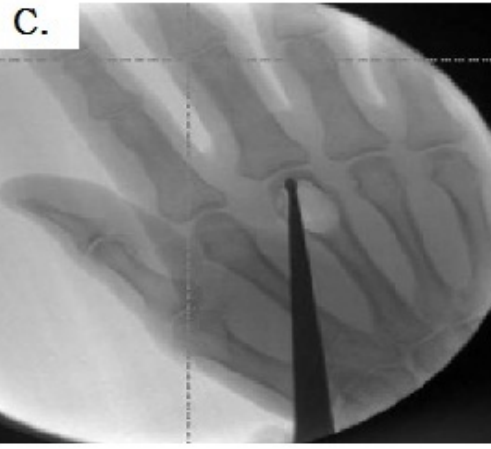

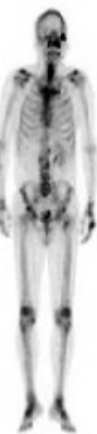
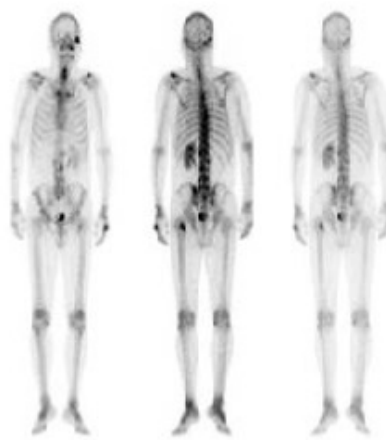

Figure 1: Imaging work-ups A) Roentgenogram of left hand showing osteolytic lesion of third metacarpal bone, B) bone scan changes from 8 year ago to the present and C) intraoperative fluoroscopy of left hand showing curettage of left third metacarpal lesion.

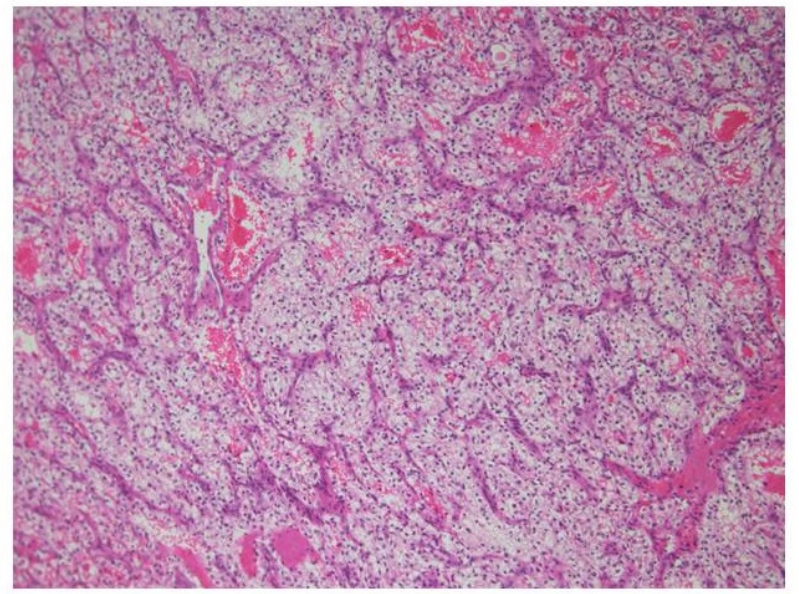

Figure 2: Histopathologic findings. Hematoxylin-eosin staining slide (original magnification $\times 100$ ); The tumor shows typical histological features of clear cell type renal cell carcinoma, clear cytoplasm surrounded by a distinct cell membrane. Regular network of small thin-walled blood vessels, a diagnostically helpful characteristics of cc-RCC, is also noted.

The underlying mechanism of the deposition of metastatic cells within the hand is currently unclear, as is the explanation of acrometastatic rarity in parts of the skeleton centers. In the past, increased vascularity and trauma have been suggested because of an increased incidence of acrometastasis in the dominant hand [6,7]. It has been suggested that the relative paucity of red marrow is the attributable cause of the rare incidence in the hands [3]. Normally, after early adult life, the small bones contain only a patch of red marrow or are entirely yellow marrow. The blood channels in fatty marrow are well formed, but in red marrow they consist of numerous thin-walled capillaries, and the rate of blood flow in red marrow is therefore decreased due to this widening of the stream bed. This would favor the deposition of tumor emboli in red marrow [7].

Acrometastasis to the hand can be asymptomatic or can present with painful swelling and movement restriction. Acrometastasis constitutes a diagnostic challenge, mainly because their clinical and radiologic presentation might be confounded with non-neoplastic conditions related to osteoarthritic disease [8]. The common differential diagnoses include acute infection; metabolic conditions like gout and several malignant or benign tumors, such as adrenocortical carcinoma, alveolar rhabdomyosarcoma, clear cell sarcoma, alveolar soft part sarcoma, malignant melanoma, fibrous dysplasia, and enchondroma $[3,9]$. These tumors should be differentiated diagnostically by histopathologists on light microscopy. In this case, before confirmation based on the surgically removed specimen of the left acrometastatic metacarpal bone, the multiple suspicious bony lesions were considered to be benign bony disease because of the previous polyostotic fibrous dysplasia at the zygoma and the absence of evident recurrence or progression on follow-up systemic work-ups, including annual or semiannual follow-up bone scans and periodic follow-up PET-CT over the course of 8 years. 
Citation: Song WH, Kim SH, Park WS, Kim SH, Kim JH, et al. (2015) Late Solitary Acrometastasis of the Hands as Unusual Metastatic Site of Renal Cell Carcinoma: A Case Report and Literature Review. J Cytol Histol S3: 027. doi:10.4172/2157-7099.S3-027

Page 3 of 3

The principle behind the treatment of acrometastasis to the hands is to aim for quick relief without adding iatrogenic complications, which present an especially important consideration in high-risk patients. Therefore, palliation of pain and movement restriction is the main clinical concern [10]. Amputation, wide excision, curettage, cementation, radiotherapy, and chemotherapy are the therapeutic options in this rare presentation [3,5,10]. A major amputation, such as one at the wrist, is usually unnecessary and would only exacerbate the patient's physical and psychogenic misery. Under most circumstances, radiation therapy, curettage, or amputation of phalanges under regional anesthesia is all that required [1].

Among patients with skeletal metastases of renal origin, approximately two-thirds have a solitary lesion, and an aggressive surgical approach has been advocated for these patients [2]. As acrometastasis is generally accompanied by widespread disease, the prognosis is poor, with an anticipated survival of 6 months, a 2-year survival rate of $50 \%$, and a 5 -year survival rate of $25 \%[2,5,11]$. However, patients with solitary metastasis diagnosed at least 12 months following nephrectomy had a 5-year survival rate after surgical treatment of osseous metastasis that was as high as 54\% [12].Therefore, this present case report suggested the importance of suspicious mind when RCC patient presented with painful lower extremities and the necessities of long-term follow-up in RCC. Identifying and effectively treating theses metastases in a timely manner can improve dramatically the patient's quality of life

\section{References}

1. Troncoso A, Ro JY, Grignon DJ, Han WS, Wexler H, et al. (1991) Renal cell carcinoma with acrometastasis: report of two cases and review of the literature. Mod Pathol 4: 66-69.
2. Mavrogenis AF, Mimidis G, Kokkalis ZT, Karampi ES, Karampela I, et al. (2014) Acrometastases. Eur J Orthop Surg Traumatol 24: 279-283.

3. Kerin R (1987) The hand in metastatic disease. J Hand Surg Am 12: 77-83.

4. Lane BR, Kattan MW (2005) Predicting outcomes in renal cell carcinoma. Curr Opin Urol 15: 289-297.

5. Stomeo D, Tulli A, Ziranu A, Perisano C, Maccauro Vde S (2015) Acrometastasis: a literature review. Eur Rev Med Pharmacol Sci 19: 2906-2915.

6. Flynn CJ, Danjoux C, Wong J, Christakis M, Rubenstein J, et al. (2008) Two cases of acrometastasis to the hands and review of the literature. Curr Oncol 15: 51-58.

7. Healey JH, Turnbull AD, Miedema B, Lane JM (1986) Acrometastases. A study of twenty-nine patients with osseous involvement of the hands and feet. J Bone Joint Surg Am 68: 743-746.

8. Salesi N, Russillo M, Marandino F, Ruggeri EM, Metro G, et al. (2007) Bone of the hands as unusual metastastatic site of renal cell carcinoma. J Exp Clin Cancer Res 26: 595-597.

9. Ünsal M, Yosma E, Keles MK, Bekci T, Fincan S, et al. (2014) Digital metastasis prior to lung adenocarcinoma diagnosis in a geriatric patient. EurGeriatr Med 6: 82-84.

10. Amadio PC, Lombardi RM (1987) Metastatic tumors of the hand. J Hand Surg Am 12: 311-316.

11. Hsu CS, Hentz VR, Yao J (2007) Tumours of the hand. Lancet Oncol 8: 157-166.

12. Dürr HR, Maier M, Pfahler M, Baur A, Refior HJ (1999) Surgical treatment of osseous metastases in patients with renal cell carcinoma. Clin Orthop Relat Res: 283-290.
This article was originally published in a special issue, entitled: "Cytopathology", Edited by Borislav A. Alexiev, University of Maryland Medical Center, USA 